\title{
Evaluación de la planta Scirpus californicus (Totora) en la eficiencia de remoción de fierro en un prototipo de humedal construido de flujo superficial
}

\section{Evaluation of the plant Scirpus californicus (Totora) efficiency removal of a prototype of iron in surface flow constructed wetland}

\author{
Cuadrado Campo, Walter Javier / Vicuña Orihuela, Carmela Emperatriz / \\ Torres Gutiérrez, Elmer Robert \\ Facultad de Ciencias Aplicadas - Tarma, Universidad Nacional del Centro del Perú
}

\section{RESUMEN}

El trabajo de investigación tiene como objetivo evaluar la eficiencia de remoción de fierro con la planta Scirpus californicus en un prototipo de humedal construido de flujo superficial, debido a que los metales afectan a las cadenas alimenticias, provocando un efecto de bioacumulación entre los organismos de la cadena trófica.

La metodología consistió en la construcción de un humedal construido de flujo superficial de $1 \mathrm{~m} \times 2 \mathrm{~m} \times 0.9 \mathrm{~m}$ cubierto con material plástico, se acondicionó 90 plantas de Scirpus californicus en 480 litros de solución de fierro.

La evaluación se realizó durante 12 días con el análisis de la raíz-rizoma, tallo sumergido y tallo aéreo previo lavado con agua destilada, además se analizó la disminución del fierro de la solución con el método de análisis por absorción atómica y el pH.

Se concluye que la remoción de fierro en la solución fue desde $5.00 \mathrm{mg} / \mathrm{l}$ hasta 2.64 $\mathrm{mg} / \mathrm{l}$ debido a la absorción de la planta con $47.20 \%$. El pH inicial fue de 3.90 el cual indica que el medio acuoso es ácido y evoluciona en forma ascendente hasta el

\section{ABSTRACT}

The research aims to assess the efficiency of iron removal plant with Scirpus californicus in a prototype surface flow constructed wetland, because the metals affect food chains, causing an effect of bioaccumulation between agencies the food chain.

The methodology involved the construction of a surface flow constructed wetland $1 \mathrm{mx} 2 \mathrm{mx}$ $0.9 \mathrm{~m}$ covered with plastic, 90 plants of Scirpus californicus was conditioned in 480 liters of solution of iron.

The evaluation was conducted for 12 days with the analysis of the root-rhizome, stem submerged and aerial stem prior washing with distilled water, further reducing the iron from solution by the method of atomic absorption analysis and the $\mathrm{pH}$ was analyzed.

It is concluded that the removal of iron in the solution was from $5.00 \mathrm{mg} / \mathrm{L}$ to $2.64 \mathrm{mg} / \mathrm{L}$ due to absorption of the plant with $47.20 \%$. The initial $\mathrm{pH}$ was 3.90 which indicates that the aqueous medium is acidic and evolves upward to the order of 4.36 indicating a tendency of the aqueous medium neutral. The absorption of iron by Scirpus californicus (reeds) plant reached the highest absorption of 
orden de 4.36 indicándonos una tendencia del medio acuoso a neutro. La absorción de fierro por la planta Scirpus californicus (totora) alcanzó la mayor absorción de fierro por la raíz-rizomas con $1684.44 \mathrm{mg} / \mathrm{kg} \mathrm{seco}$, seguido por el tallo sumergido $325.14 \mathrm{mg} /$ $\mathrm{kg}$ seco y en menor cantidad en el tallo aéreo con $171.30 \mathrm{mg} / \mathrm{kg}$ seco.

Palabras clave: fitorremediación, remoción de fierro, Scirpus californicus, contaminación ambiental. iron by the root-rhizome with $1684.44 \mathrm{mg}$ / kg dry, followed by the stem submerged $325.14 \mathrm{mg} / \mathrm{kg}$ dry and fewer in the aerial stem with $171,30 \mathrm{mg} / \mathrm{kg}$ dry.

Keywords: phytoremediation, removal of iron, Scirpus californicus, environmental pollution.

\section{INTRODUCCIÓN}

La contaminación en los ríos se produce por presencia de compuestos o elementos que normalmente no se encontrarían sin la acción del hombre, o por un aumento en la concentración normal de las sustancias ya existentes. Uno de los contaminantes potencialmente más tóxicos son los metales pesados, el aporte de estos al ciclo hidrológico procede de dos fuentes, una de origen litogénico o geoquímica a partir de los minerales que por causas de erosión, lluvias, etc., son arrastradas al agua y otra de origen antropogénico o debida a la actividad humana. De las diferentes sustancias existentes, más de un millón han sido introducidas en las aguas naturales a través de vertidos antropogénicos (Förstner et al., 1993).

El estudio de los metales pesados radica su importancia en las características que estos presentan, tales como: su alta persistencia, rápida acumulación por los organismos vivos, efectos difícilmente detectables a corto plazo, y su elevada toxicidad, entre otras. Por lo general, la toxicidad de los metales pesados es proporcional a la facilidad de ser absorbidos por los seres vivos, del estado de oxidación en que se encuentre el metal, de su capacidad de enlace con otros compuestos o de su interacción con los ácidos nucleicos, proteínas y enzimas, que responden a funciones celulares, produciendo alteraciones en los tejidos y órganos de los seres vivos. Por otra parte, estos son utilizados como indicadores de contaminación ambiental, ya que, su presencia en algas, moluscos o peces, es usada para establecer el grado de contaminación del ambiente acuático (Sánchez, 2010) Los metales pesados son referidos como "metales trazas", por presentarse a bajas concentraciones (1 ppm o menos). Generalmente muchos de los metales que se encuentran en cantidades traza, son esenciales para la vida de algunos organismos, dentro de estos se incluyen: aluminio, cromo, cobre, hierro, manganeso, molibdeno, níquel, selenio, vanadio y zinc (Contreras, 2006).

En la Actualidad, los metales pesados tienen un gran significado como indicadores de la calidad ecológica de todo flujo de agua debido a su toxicidad y especialmente al comportamiento bioacumulativo que estos poseen (Aguilar y Sagástegui (2014). Una de las macrófitas más conocidas y difundidas en nuestro medio es la Totora (Scirpus californicus). Esta especie fue traída del Lago Titicaca a la Laguna Alalay, donde forma parte de la flora presente en la laguna y también se encuentra en abundancia en las zonas de inundación de la planta de tratamiento de Alba Rancho. Esta macrófita, forma parte de la amplia gama de plantas fitodepuradoras empleadas en los sistemas no convencionales de depuración de aguas residuales.

Dentro de la región Junín especialmente para la provincia de Tarma, tiene una gran importancia, realizar el estudio de las plantas nativas scirpus californicus en la remoción de metales pesados y conocimiento como una alternativa de descontaminación de las aguas. 
Por esta razón, se plantea la siguiente hipótesis: La planta Scirpus californicus (totora) es considerado como una alternativa para la depuración de soluciones de fierro mediante un prototipo de humedal construido de flujo superficial.

El objetivo General: Evaluar la eficiencia de remoción de fierro con la planta nativa Scirpus californicus en un prototipo de humedal construido de flujo superficial.

Los objetivos específicos:

> Construir el prototipo de humedal construido de flujo superficial

> Determinar la eficiencia de remoción de fierro de la solución.

> Evaluar el comportamiento del pH frente al tiempo para su evaluación del tratamiento.

$>$ Determinar la absorción de fierro por la raíz-rizomas, tallo sumergido y tallo aéreo de la planta scirpus californicus frente al tiempo.

\section{MATERIALES Y MÉTODOS}

Lugar de Ejecución

La presente investigación se realizó en la Facultad de Ciencias Aplicadas, Laboratorio de Química Analítica y del Ambiente de la Universidad Nacional del Centro del Perú. Ubicada a 3050 msnm en la comunidad de Pomachaca anexo del distrito de Tarma - Junín, km 5 carretera central a Chanchamayo, la temperatura se encuentra entre 15 y $20^{\circ} \mathrm{C}$.

Población: Las plantas Scirpus californicus (totora) de la comunidad de Pomachaca - Tarma.

Muestra: Lo constituye las partes de la planta raiz-rizomas, tallo sumergido, tallo aéreo.

Materiales:

20 vasos de precipitación de $150 \mathrm{ml}$

20 fiolas de vidrio de $100 \mathrm{~mL}$

20 lunas de reloj

20 embudos de vidrio

01 pipeta volumétrica de $2 \mathrm{~mL}$

01 pipeta volumétrica de $5 \mathrm{~mL}$

01 pipeta volumétrica de $10 \mathrm{~mL}$

02 probetas de $100 \mathrm{~mL}$

02 cajas de 100 papel de filtro cuantitativo
Equipos: 01 Estufa Marca MEMMERT ALEMAN, 01 Molino Universal Marca IKA M20, 01 pH-metro Marca HANNA 3220, 01 Espectrofotómetro Marca VARIAN 240, 01 Balanza analítica Marca E. METTLER,

Reactivos Para Análisis Físico Químico

Acido nítrico, ácido clorhídrido, acido perclóri$\mathrm{co}$, Sales de nitrato de fierro.

\section{Método de Investigación}

El método de investigación a realizarse en el presente trabajo es del tipo aplicativo. El trabajo tiene carácter experimental porque se va manipular a la planta a diferentes tiempos de tratamiento para relacionar con la realidad.

\section{Prueba Estadística}

Se realizará con el software Excel para mostrar gráficamente la eficiencia y comportamiento de la planta frente al tiempo.

Figura 1. Diseño estadístico de la investigación

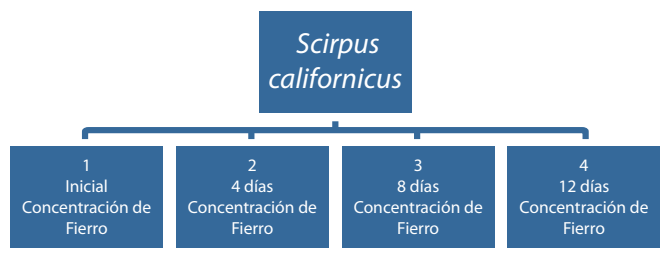

\section{Tratamientos:}

T1: Concentración de fierro en la raíz-rizoma, tallo sumergido y tallo aéreo al inicio

T2: Concentración de fierro en la raíz-rizoma, tallo sumergido y tallo aéreo a los 4 días con solución de fierro.

T3: Concentración de fierro en la raíz-rizoma, tallo sumergido y tallo (aéreo) a los 8 días con solución de fierro.

T4: Concentración de fierro en la raíz-rizoma, tallo sumergido y tallo aéreo los 12 días con solución de fierro.

\section{Metodología de la experimentación:}

Para el presente trabajo de investigación se realizó los siguientes pasos:

Construcción del prototipo de humedal construido de flujo superficial.

Obtención de las plantas Scirpus californicus (totora) de los alrededores de Pomachaca -Tarma. 
Análisis de fierro de la planta

Acondicionamiento de la planta Scirpus californicus en el humedal con solución de fierro de $5 \mathrm{mg} / \mathrm{L}$

Análisis de fierro de la raíz-rizoma, tallo sumergido y tallo aéreo a diferentes días.

Flujograma para medir la absorción de fierro por la planta.

\section{Extracción}

La planta Scirpus californicus se ha extraído de los alrededores del lugar de investigación cuidando que no se maltrate la planta.

\section{Selección}

La selección de la planta pasó por una inspección visual, tomando en cuenta que la planta tenga el tamaño y buen estado físico raíz-rizoma, tallo sumergido y tallo aéreo para poder vivir.

\section{Lavado 1}

El primer lavado permitió eliminar las impurezas que acompañan a la planta como tierra, piedras, etc. hasta que el agua sea clara y libre de solidos

\section{Lavado 2}

El segundo lavado se realizó con agua destilada para eliminar las impurezas que pueden permitir la precipitación de fierro.

\section{Acondicionamiento}

Las plantas se acondicionaron en el tanque de concreto protegido con material plástico de $1 \mathrm{~m}$ x $2 \mathrm{~m} \times 0.9 \mathrm{~m}$ en 480 litros de solución de 5.0 $\mathrm{mg} / \mathrm{L}$ de fierro.

\section{Muestreo}

El muestreo se realizó por triplicado al inicio de la pruebas, luego cada 4 días hasta los 12 días. Las muestras se dividieron en raíz-rizoma, tallo sumergido y tallo aéreo luego se lavó 4 veces con agua potable y 2 veces con agua destilada para eliminar cualquier contaminación en las superficies de la planta.

\section{Secado}

Las muestras lavadas con agua destilada oreadas se acondicionaron en mallas y luego se secaron a $60^{\circ} \mathrm{C}$ por 80 horas.

\section{Análisis}

Los análisis foliares se realizaron utilizando el método normalizado por absorción atómica.

La metodología de absorción es según el diagrama de flujo propuesto para fierro (Figura 2).

Figura 2 Diagrama de flujo de absorción de fierro

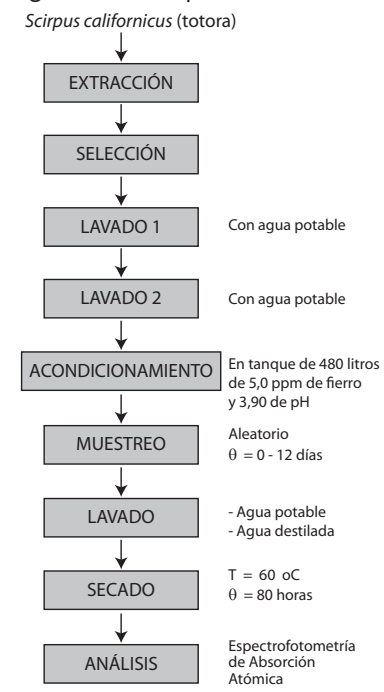

\section{RESULTADOS}

Evaluación de la planta scirpus californicus (totora) en la eficiencia de remoción de fierro.

\section{Construcción del prototipo de humedal cons- truido de flujo superficial.}

Figura 3. Humedal construido de flujo superficial

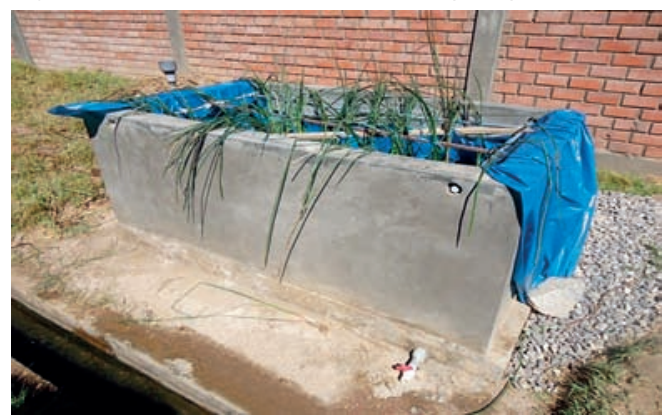


Determinación de la eficiencia de remoción de fierro de la solución.

La tabla 1 presenta la disminución de concentración de fierro de la solución durante los 12 días.

Tabla 1. Variación de la concentración de fierro de la solución.

\begin{tabular}{ccccc}
\hline Días & 0 & 4 & 8 & 12 \\
\hline $\begin{array}{c}\text { Concentración } \\
\text { de fierro }(\mathrm{mg} / \mathrm{L})\end{array}$ & 5 & 4.12 & 3.5 & 2.64 \\
\hline
\end{tabular}

Figura 4. Absorción de fierro frente al tiempo.

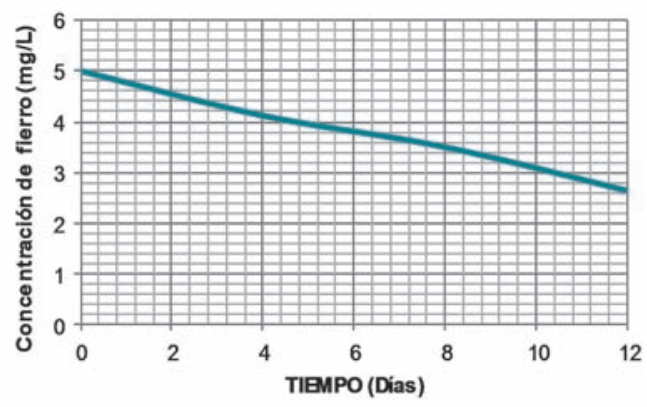

\section{Comportamiento del $\mathrm{pH}$ frente al tiempo}

En la figura 5 se muestra el pH de la solución de fierro.

Figura 5. Comportamiento del pH frente al tiempo en la solución de fierro.

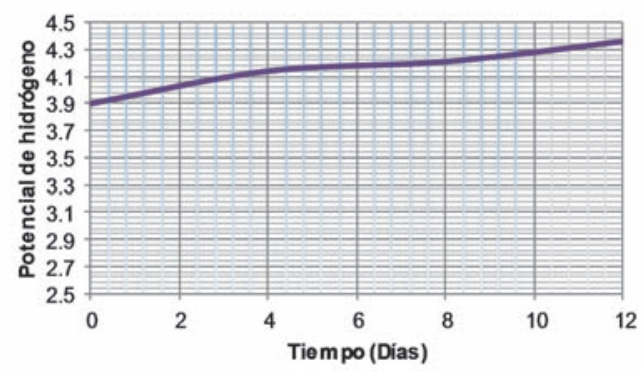

Determinación de la absorción de fierro por la raíz-rizomas, tallo sumergido y tallos aéreo de la planta Scirpus californicus frente al tiempo.

Figura 6. Absorción de fierro en la raíz-rizoma, tallo sumergido y tallo aéreo de la planta Scirpus californicus (totora) frente al tiempo.

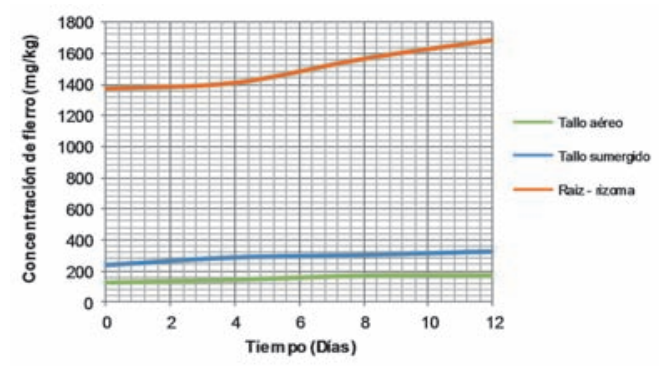

\section{DISCUSIÓN}

Construcción del prototipo de humedal construido de flujo superficial.

En la figura 3 se muestra el humedal construido de flujo superficial con la planta scirpus californicus(totora) el cual se construyó de ladrillo revestido con concreto y cubierto con material plástico cuyas dimensiones es de $1 \mathrm{~m} \times 2 \mathrm{~m} \times$ $0.9 \mathrm{~m}$. De acuerdo a la investigación realizado por Serrano, (2005). Se preparó 480 litros de solución ácida de $5.0 \mathrm{ppm}$ de fierro con agua sin tratar del lugar y se acondicionó 90 plantas de Scirpus californicus para su evaluación.

Determinación de la eficiencia de remoción de fierro de la solución.

En la tabla 1 y figura 4, se muestran los resultados de la eficiencia de remoción de fierro de la solución. Durante los 12 días que se evaluó el fierro de la solución la remoción fue desde $5.00 \mathrm{mg} / \mathrm{l}$ hasta $2.64 \mathrm{mg} / \mathrm{l}$ debido a la absorción de la planta scirpus californicus a través de su raíz- rizoma y tallo. Similar a lo desarrollado por Serrano (2005) en su trabajo de investigación de tesis para maestría con la planta typha angustifolia donde la remoción fue 
desde $0.3172 \mathrm{mg} / \mathrm{l}$ a $0.1208 \mathrm{mg} / \mathrm{L}$ de fierro de las aguas de mina en 4 días.

\section{Comportamiento del $\mathrm{pH}$ frente al tiempo.}

En la figura 5, se muestra los resultados del comportamiento del potencial de hidrógeno donde el $\mathrm{pH}$ inicial del orden de 3.90 indica que el medio acuoso es ácido. Podemos apreciar que el $\mathrm{pH}$ durante los 12 días evoluciona en forma ascendente hasta el orden de 4.36 indicándonos una tendencia del medio acuoso a neutro. El pH se ha incrementado similar a lo obtenido por (Serrano, 2005) donde pH inicial fue de 5 y aumento a 6.5.

\section{Determinación de la absorción de fierro por la raíz-rizomas, tallo frente al tiempo.}

En la figura 6, se muestra los resultados de la absorción de fierro por la planta Scirpus californicus(totora) alcanzando la mayor absorción de fierro en 12 días por la raíz-rizomas con $1684.44 \mathrm{mg} / \mathrm{kg} \mathrm{seco}$, seguido por el tallo sumergido $325.14 \mathrm{mg} / \mathrm{kg}$ seco y en menor cantidad en el tallo aéreo con $171.30 \mathrm{mg} / \mathrm{kg}$ seco el cual se sustenta según ( Raskyn \& Smith, 2000): La presencia de altas concentraciones de metales en Scirpus californicus (caso de hierro, manganeso y aluminio) indicaría que esta planta es bioacumuladora. Es importante mencionar que la bioacumulación no es un proceso adverso sino natural en el desarrollo de las plantas. También según (Kabata-pendias, 2011): Las especies vegetales, incluidos algunos cultivos, tienen la capacidad de acumular metales en sus tejidos.

\section{CONCLUSIONES}

Mediante el empleo de humedal de flujo superficial se logró remover la acidez y concentración de fierro de la solución de $5.0 \mathrm{mg} / \mathrm{l}$ utilizando 90 plantas scirpus californicus (totora) en la facultad de ciencias aplicadas de la Universidad Nacional del Centro del Perú ubicada en la provincia de Tarma anexo pomachaca, Perú.

Al término de los 12 días de tratamiento se logró remover el pH de 3.90 a 4.36 .
El porcentaje de remoción de fierro de la solución fue de $47.20 \%$.

Se determinó la absorción de fierro por la planta scirpus californicus aumentando su concentración de fierro inicial en sus partes alcanzando valores en la raíz-rizomas de 1372.31 a $1684.44 \mathrm{mg} / \mathrm{kg}$, tallo sumergido de 237.22 a $325.14 \mathrm{mg} / \mathrm{kg}$, tallo aéreo de 125,34 a 171.30 $\mathrm{mg} / \mathrm{kg}$ seco de muestra.

\section{RECOMENDACIONES}

- Realizar otros estudios científicos tecnológicos para determinar la remoción y absorción de otras plantas acuáticas de metales pesados que afectan la cadena alimenticia.

- Es recomendable hacer más estudios sobre evaluación y mecanismos de remoción de metales pesados por plantas acuáticas.

- Realizar más trabajos de investigación para industrializar en forma integral la totora, con el aprovechamiento integral del tratamiento de las aguas.

\section{REFERENCIAS BIBLIOGRÁFICAS}

1. Aguilar, L. \& Sagástegui, S.(2014). Capacidad acumuladora de cadmio en raíces de Scirpus californicus expuestas a diferentes concentraciones de nitrato de cadmio en condiciones de laboratorio. Revista REBIOLEST, 1(2), 14-22. Recuperado de file://C:/Users/PC17/Downloads/472956-1-PB.pdf

2. APHA (1998). Standard methods for the examination of water and wastewater. (20th Ed.) American Public Health Association. Washington. Recuperado de https://www. standard methods.org/

3. Baker, J. \& Brooks, R. (1989).Terrestrial higher plants which hyperaccumulate metallic elements: A review of their distribution, ecology and phytochemistry. Biorecovery 1: 81-126. Recuperado de http://www.ecoweb.com/edi/090227.html

4. Contreras, S.(2006). Determinación de metales traza (Ni, $\mathrm{Cr}, \mathrm{Cd}, \mathrm{Fe}, \mathrm{Mg}$, Al) en totora (Scirpus californicus) y sedimento en el san- 
tuario de la naturaleza Carlos Anwandter, río Cruces, Valdivia. Tesis de Químico Farmacéutico de la Universidad Austral de Chile. Recuperado de http://cybertesis.uach.cl/ tesis/uach/2006/fcc764d/doc/fcc764d.pdf

5. Kabata-pendias, A. (2011). Trace elements in soils and plants. Fourth edition. CRC Press,-Boca Raton, USA. 467 p. Recuperado de http://www.petronet.ir/documents/10180/2323242/Trace_Elements_ in_Soils_and_Plants.

6. Lara, J. (1999). Depuración de aguas residuales urbanas mediante humedales artificiales. (tesis de maestría) Universidad Politécnica de Cataluña -Instituto Catalán de Tecnología, Barcelona. Recuperado de http://www.aprchile.cl/pdfs/Humedales.pdf

7. Moreno, E. (2010). Recuperación de suelos mineros contaminados con arsénico mediante fitotecnologías, (tesis de doctorado), Universidad Autónoma de Madrid, España. Recuperado de http://www.uam.es/personal_pdi/ciencias/emjimene/Publicaciones/ PhD_Thesis_EMorenoHQ.pdf

8. Mufarrege, G. (2012). Tolerancia y eficiencia de Typha domingensis Pers. en la retención de metales y nutrientes de efluentes industriales. (tesis de doctorado). Universidad Nacional del Litoral, Argentina. Recuperado de http://bibliotecavirtual.unl.edu. ar:8180/tesis/bitstream/1/406/1/Tesis.pdf Raskyn, I.y Smith, R. (2000). Phytoremediation of toxic metals: Using plants to clean up the environment. 304p. Recuperado de http://www.wiley.com/WileyCDA/WileyTitle/productCd-0471192546.html.

9. Sanchez, G., (2010). Mecanismos de adsorción y acumulación intracelular de $\mathrm{Pb}^{+2}$ en salvinia mínima, (tesis de doctorado), Universidad Autónoma Metropolitana - México. Recuperado de http://wwwl inecol.edu. $\mathrm{mx} / \mathrm{cv} / \mathrm{CV}$ _pdf/CV_Eugenia_Olguin_21_ feb_2013.pdf

10. Serrano, C. (2005). Tratamiento del drenaje acido de la mina Sipan - distrito de Llapa Cajamarca - mediante humedales artificiales de flujo superficial.( tesis de maestría) UNT. Trujillo - Perú.

11. Villarroel, C., (2005). Tratamiento terciario del efluente de la planta de tratamiento de aguas residuales el Cortijo para uso agrícola con humedales construidos de flujo superfi- cial - Trujillo, (tesis de doctorado), Universidad Nacional de Trujillo. Recuperado de http://www.ciiq.org/varios/peru_2005/Trabajos/III/3/3.3.15.pdf. 